\title{
Les spectromètres à temps de vol
}

\author{
H. Casalta
}

Institut Laue-Langevin, 6 rue Jules Horowitz, BP. 156, 38042 Grenoble cedex 9, France

\begin{abstract}
Résumé. Ce cours porte plus particulièrement sur les spectromètres de type «temps de vol » construits en France auprès de sources de neutrons continues, c'est à dire au réacteur Orphée (Saclay) et à l'Institut Laue-Langevin (Grenoble)
\end{abstract}

\section{PRINCIPE DU SPECTROMÈTRE DE "TEMPS-DE-VOL"}

Les spectromètres de type "temps-de-vol" sont utilisés pour des mesures inélastiques de diffusion des neutrons, c'est à dire pour déterminer un changement d'énergie du neutron lors de son interaction avec le systeme étudié. On note $E_{i}$ et $E_{f}$ les énergies initiale et finale du neutron et $\hbar \omega=E_{i}-E_{f}$ le transfert d'énergie. La détermination de $\hbar \omega$ se fait via la connaissance de $E_{i}$ et $E_{f}$; Différentes méthodes sont utilisées pour déterminer ou selectionner l'énergie du neutron:

- La méthode de "temps-de-vol" consiste à mesurer le temps mis par le neutron pour parcourir une distance donnée. La vitesse du neutron $V_{n}$ est typiquement de l'ordre du $\mathrm{km} / \mathrm{sec}$.

$$
\lambda(\AA) \times V_{n}(\mathrm{~km} / \mathrm{s})=3.95603
$$

Cela signifie que sur une distance de quelques metres avec une résolution en temps de quelques dizaines de $\mu$ sec., on peut déterminer $V_{n}$ à quelques $\%$ près $\left(d V_{n} / V_{n} \sim q q \%\right)$. Pour mesurer un temps à l'aide d'une horloge, il est nécessaire de définir un temps $t=0$ et concrètement de "hacher" le faisceau de neutrons en bouffées successives. Dans le cas d'une source pulsé comme ISIS en Angleterre, il n'y a rien à faire, mais dans le cas d'un réacteur traditionnel comme à Saclay ou à l'ILL, cela nécessite l'utilisation de "choppers".

- Une autre méthode consiste à diffracter le faisceau de neutrons par un cristal. L'énergie du neutron se déduit par la loi de Bragg:

$$
n \lambda=2 d \sin \theta
$$

où $\mathrm{n}$ est un entier, $\lambda$ la longueur d'onde du neutron considéré, $d$ la distance inter-réticulaire et $\theta$ représente l'angle de diffusion. Pour une orientation fixée du cristal, l'angle $\theta$ correspond à une valeur unique de $\lambda$ (aux ordres superieures $\lambda / n$ près).

Les spectromètres de "temps-de-vol" construits auprès de sources de neutrons continues (Orphee ou l'ILL) utilisent une géométrie dite directe. En amont de l'échantillon (partie primaire), une bouffée monochromatique est créee (neutron de vitesse initiale $V_{i}$ connue) en utilisant une des méthodes présentées ci-dessus. Apres diffusion par l'échantillon, le neutron possede une vitesse finale $V_{f}$ qui est analysée sur une certaine base de temps et dont la valeur dépend du processus mis en jeu lors de la diffusion. Cette partie en aval de l'échantillon constitue le spectrometre secondaire.

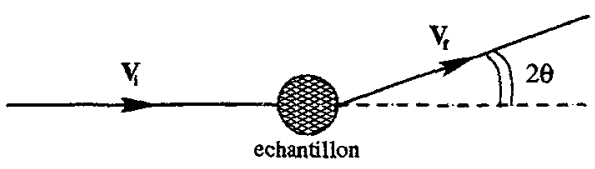

\section{1 spectromètre primaire}

La création d'une bouffée monochromatique est téalisée en utilisant un système constitué uniquement de choppers ou un système mixte de choppers et de cristal monochromateur. Nous verrons ces différentes méthodes à travers la présentation de spectromètres existants (section 2). Parallelement à la création d'une 


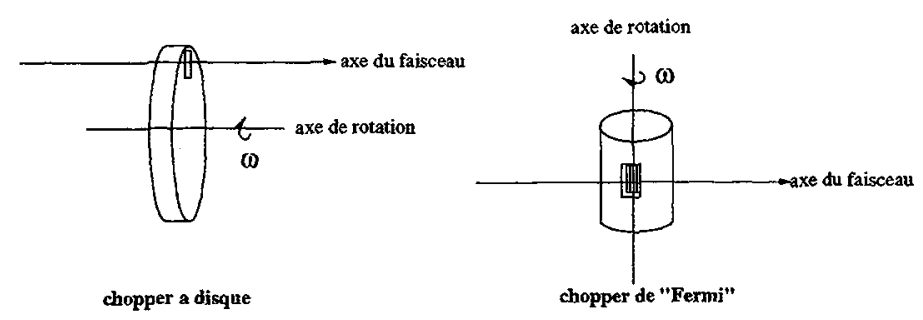

Figure 1: Choppers.

bouffée, un signal électrique est généré de manière à synchroniser la détection électronique (réfèrence de temps $t=0$ ).

Il existe differents types de choppers, mais ce n'est pas le but de ce cours de donner trop de détails techniques à ce sujet. Le type le plus simple consiste en un chopper à disque recouvert d'un materiau absorbant pour les neutrons, tournant suivant l'axe du faisceau et presentant une ou plusieurs fente(s) (voir figure 1). La vitesse angulaire $\omega$, le rayon $R$ et la largeur de la fente $d$ du chopper sont les parametres caractéristiques qui interviennent dans la création d'une bouffée. Si la largeur du faisceau correspond à la largeur de la fente $d$, la fonction de transmission en temps du chopper est de forme triangulaire avec une largeur à mi-hauteur $\Delta t$ donnée par la relation suivante:

$$
\Delta t=\frac{d}{R \omega}
$$

$\Delta t$ représente le temps d'ouverture du chopper et constitue un des parametres du "temps-de-vol". II est à noter que si la fente du chopper est plus large que le faisceau, la fonction de transmission en temps a une forme trapèzoïdale.

Il est important de réaliser qu'une grande partie du flux de neutrons est "perdue" au passage d'un chopper dans le cas d'une source continue. En effet dans le cas d'un chopper à disque, la fraction utilisée est donné par le rapport entre l'ouverture de la fente et la circonférence du disque. Pour un chopper à $\mathbf{n}$ fentes, on a donc une perte de:

$$
\text { perte }=1-\frac{n d}{2 \pi R}
$$

application numérique: $n=1, d=2 \mathrm{~cm}, R=24 \mathrm{~cm}$, donne une perte de flux de $98.7 \%$. Ceci constitue un des inconvénients techniques du "temps-de-vol" sur source continue. Dans la comparaison avec les spectromètres de type “3-axes”, cette perte d'intensité est compensée par la détection des neutrons diffusés sur un angle solide beaucoup plus important et par la mesure simultanée de tous les transferts d'énergie.

Il existe un autre type de chopper communément utilisé dit de "Fermi". Ce type de chopper se présente comme un tambourin fendu dans le sens de la tranche contenant un collimateur et dont l'axe de rotation est perpendiculaire au faisceau de neutrons (voir figure 1).

\section{2 spectromètre secondaire}

La distance entre l'échantillon et les détecteurs est typiquement de quelques mètres permettant une déterminati précise de $V_{f}$ (via la connaissance du temps mis par le neutron pour parcourir cette distance). Cette distance représente la longueur de vol notée $L$. Elle doit être suffisamment grande pour avoir une bonne résolution en temps et donc en énergie, tout en ne réduisant pas trop l'angle solide de détection.

La détection se fait avec un certain retard $t_{0}$ par rapport au temps $t=0$ définit par rapport à l'un des choppers. Les neutrons sont détectés sur une certaine base de temps divisés en $N$ canaux d'intervalle $\delta t$. La base de temps s'inscrit dans le laps de temps separant deux bouffees successives. 
La détection est effectuée sur une surface importante (quelques $m^{2}$ ) à l'aide de tubes remplis $d^{\prime} \mathrm{He}^{3}$ ou d'un multi-détecteur. tives:

Le tableau suivant récapitule les principaux paramètres du "temps-de-vol" et leurs influences respec-

\begin{tabular}{|l|l|c|} 
paramètre & influence & notation \\
\hline yitesse incidente & flux, résolution, domaine en $Q$ & $\mathrm{~V}_{i}$ \\
largeur de fente & flux (sur l'échantillon), résolution & $\mathrm{d}$ \\
vitesse de rotation & taux de répétition, résolution & $\omega$ \\
longueur de vol & résolution, angle solide & $\mathrm{L}$ \\
largeur des canaux en temps & (résolution) & $\delta \mathrm{t}$ \\
retard & intervalle d'analyse & $\mathrm{t}_{0}$ \\
nombre de canaux en temps & durée d'analyse, taux de répétition & $\mathrm{N}$
\end{tabular}

\section{3 particularités de la mesure en "temps-de-vol"}

- La mesure n'est pas faite a $Q$ constant. On note $\overrightarrow{k_{i}}$ et $\overrightarrow{k_{f}}$ les vecteurs d'ondes initial et final du neutron, $2 \theta=$ angle $\left(\overrightarrow{k_{i}}, \overrightarrow{k_{f}}\right)$ l'angle entre ces deux vecteurs et $\vec{Q}$ le vecteur de diffusion:

$$
\vec{Q}=\overrightarrow{k_{i}}-\overrightarrow{k_{f}}
$$

En utilisant le fait que $E=\frac{\hbar^{2} k^{2}}{2 m_{n}}$ et $\hbar \omega=E_{i}-E_{f}$, on peut écrire:

$$
\frac{\hbar^{2} Q^{2}}{2 m_{n}}=2 E_{i}-\hbar \omega-2 E_{i} \sqrt{1-\frac{\hbar \omega}{E_{i}}} \cos 2 \theta
$$

La mesure est faite à angle $2 \theta$ fixé. En géométrie directe $E_{i}$ est une constante, et l'on voit à travers l'équation (6) que dans un même détecteur (dont la position est définie par $2 \theta$ ), on collecte des neutrons diffusés avec différentes valeurs de $Q$, suivant la valeur de $\omega$.

- La mesure se fait en temps alors que l'on s'intéresse à un spectre en énergie. Il suffit de convertir de temps à énergie et cette conversion est non linéaire du fait de la relation liant $E$ et $V$ :

$$
E=\frac{1}{2} m_{n} V^{2}=\frac{1}{2} m_{n}\left(\frac{L}{t}\right)^{2}
$$

où $m_{n}$ est la masse du neutron et $t$ le temps mis pour parcourir la distance $L$. Le facteur de structure dynamique $S(Q, \omega)$ est relie à la section efficace de diffusion par la relation suivante $(d \Omega$ : angle solide de diffusion):

$$
S(Q, \omega) \sim \frac{k_{i}}{k_{f}} \frac{d^{2} \sigma}{d \Omega d E} \sim \frac{k_{i}}{k_{f}} \frac{d t}{d E} \frac{d^{2} \sigma}{d \Omega d t} \sim \frac{1}{t^{4}} \frac{d^{2} \sigma}{d \Omega d t}
$$

En effet $k_{i}$ est constant et $k_{f}$ varie comme $V_{f}$ en $1 / t$ dans une géométrie directe. Ceci a pour effet de déformer le spectre lors de la conversion de temps à énergie.

Les spectrometres actuels sont équipés de programme de traitement de données qui permettent une visualisation rapide de $S(Q, \omega)$ durant le déroulement de l'expérience.

\section{4 diagramme en temps}

Une façon simple de visualiser une expérience de "temps-de-vol" consiste à tracer un diagramme avec le temps en abscisse et la distance parcourue par le neutron en ordonnée: 


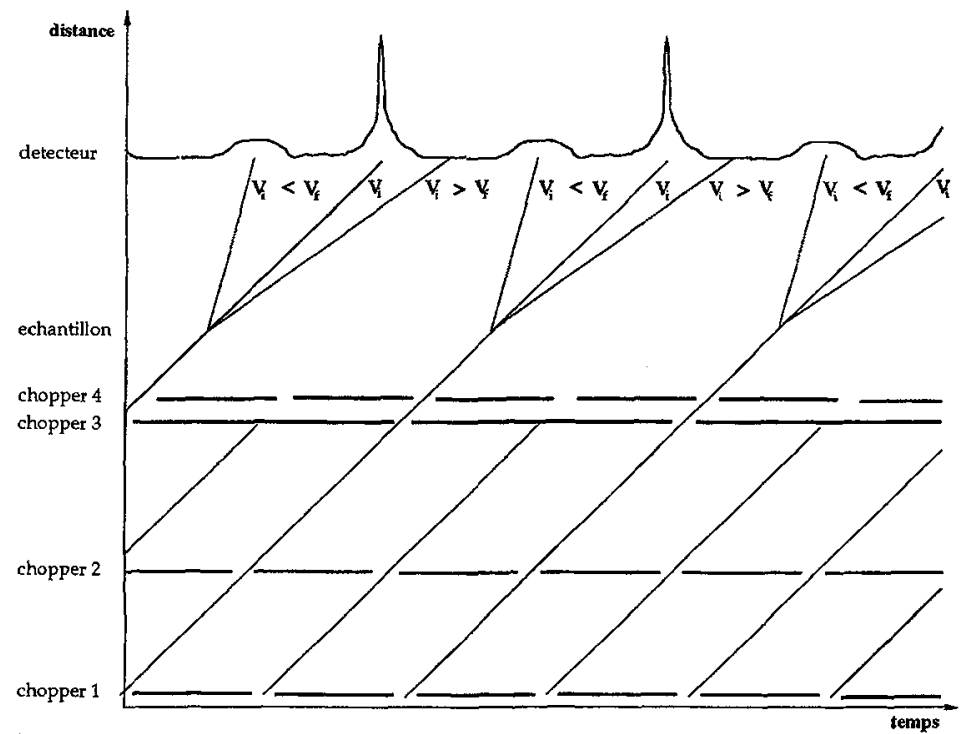

Figure 2: représentation schématique d'une expérience de temps de vol dans le cas d'un spectromètre primaire consistué de 4 choppers.

\section{INSTRUMENTS EXISTANTS À L'ILL ET À ORPHÉE}

Pour plus de détails techniques sur chacun des instruments présentés nous recommandons de se réferrer aux sites web respectifs de 1'ILL www.ill.fr et du Laboratoire Léon Brillouin www-llb.cea.fr.

\section{1 spectromètre IN5 (ILL)}

Le spectromètre IN5 est construit en sortie d'une source froide et dispose d'une distribution de longueur d'onde continue centree autour de $\lambda=4 \AA\left(E_{i}=5.1 \mathrm{meV}\right)$ s'étalant typiquement de 1.8 à $20 \AA$.

Le spectrometre primaire comprend 4 choppers à disque de diamètre $52 \mathrm{~cm}$ tournant suivant l'axe du faisceau. Chaque chopper est recouvert d'oxyde de Gadolinium (matériau absorbant pour les neutrons) et possede une seule fente de largeur à mi-hauteur $d=2 \mathrm{~cm}$ (sauf le second chopper qui a une fente de $d=4$ $\mathrm{cm}$ ). Ces 4 choppers sont suspendus magnétiquement et tournent en règle générale à une vitesse angulaire de $\omega=20000$ r.p.m. (le troisième chopper tourne éventuellement à des vitesses plus faibles). Le principe du spectrometre primaire est le suivant:

- Les choppers 1 et 4 jouent le rôle de sélecteur et produisent une bouffée de forme triangulaire en temps. La selection en longueur d'onde se fait via le déphasage entre les fentes du premier et du quatrième chopper. Ce déphasage est defini à $2 p \pi$ près et des neutrons plus lents ou plus rapides peuvent aussi passer (harmoniques).

- le second chopper filtre ces harmoniques. En interposant un troisième chopper on définit la phase de sélection de manière unique.

- le troisieme chopper permet éventuellement d'éliminer une certaine fraction des bouffées de façon a analyser le spectre diffusé sur une base de temps plus longue. Il tourne à une vitesse angulaire de $\omega / \mathrm{N}$, où $\mathrm{N}$ est le ratio (nombre entier). On accumule habituellement les données en temps durant la période séparant deux bouffées successives. Si cette période est trop courte, il y a un risque de recouvrement des différentes périodes d'analyse dû à des neutrons trop rapides qui peuvent venir contaminer les données de la période précèdente.

La détection est assurée aux petits angles de diffusion par un multi-détecteur et dans la gamme 11 à $126 \mathrm{deg}$. par des tubes à $\mathrm{He}^{3}$. Les détecteurs sont placés à $4 \mathrm{~m}$ de l'échantillon. La détection se fait sur une base de temps de $3 \mathrm{~N}$ msec. (pour $\omega=20000$ r.p.m.) et les données sont digitalisés sur 511 canaux. La résolution en énergie varie comme $1 / \lambda^{3}$ et est typiquement de $200 \mu \mathrm{eV}$ pour $\lambda=4 \AA$. 


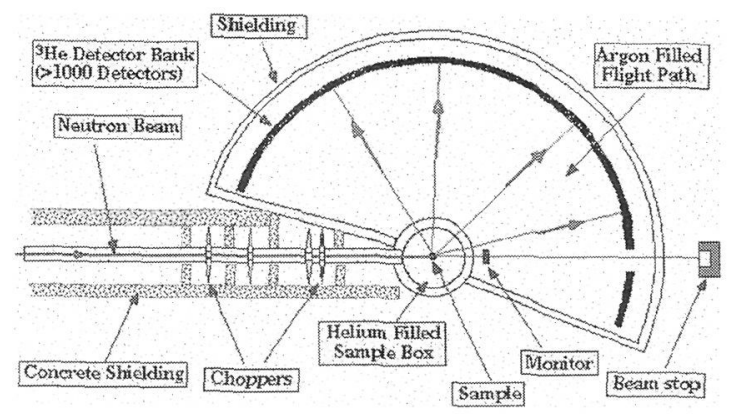

Figure 3: Spectromètre IN5.

\section{2 spectromètre Mibemol (Orphée)}

Le spectromètre Mibemol est implanté à l'extrémité d'un guide de neutrons froids et dispose d'une distribution de longueurs d'onde continue entre 2 et $10 \AA$ maximum autour de $5 \AA$

Le principe de fonctionnement de la partie primaire se rapproche de celui d'IN5, à la différence près que les choppers 1 et 4 ont été remplacés par des couples de 2 choppers tournant en sens opposé (choppers en contre-rotation). Les choppers en contre-rotation permettent d'obtenir une bouffée de même caractérisques que celle d'un chopper à disque unique tout en utilisant une vitesse de rotation $\omega$ plus faible. Ceci est important dans la mesure où la vitesse de rotation est l'un des facteurs limitants dans le développement de nouveaux instruments (problème de résistance des matériaux tournants à grande vitesse).

La base de "temps-de-vol" est constituée d'une enceinte remplie d'Hélium (pour éviter la diffusion par 1'air). La distance entre l'échantillon et les détecteurs est de $3.58 \mathrm{~m}$. La gamme en angle de diffusion va de $12 \mathrm{a} 147 \mathrm{deg}$.

Mibemol est optimisé pour mesurer des transferts d'énergie compris entre 0.01 et $10 \mathrm{meV}$. Ses performances sont comparables à celles d'IN5.

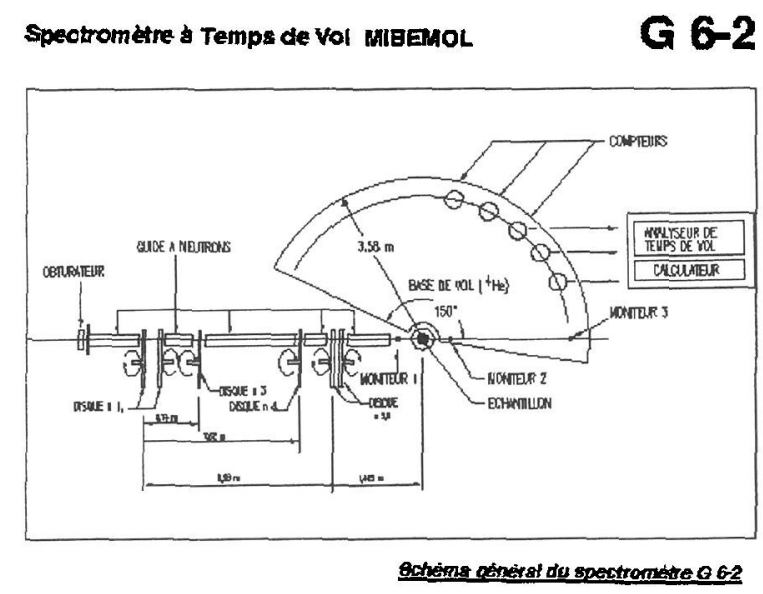

Figure 4: Spectromètre mibemol.

\section{3 spectromètre IN6 (ILL)}

Le spectromètre IN6 est base sur le principe de "focalisation en temps" du faisceau de neutrons qui lui permet d'avoir un flux plus important. IN6 est également sur source froide mais n'utilise qu'un nombre limite de longueur d'onde $(\lambda=4.1,4.6,5.1,5.9 \AA)$. 
Le spectrometre primaire comprend 3 cristaux monochromateurs en graphite pyrolitique, un filtre au Beryllium qui élimine les neutrons rapides et deux choppers de type Fermi. Le principe de focalisation en temps est le suivant: les trois monochromateurs sont alignés de façon à sélectionner respectivement les longueurs d'onde $\lambda_{0}-\delta \lambda_{0}, \lambda_{0}$ et $\lambda_{0}+\delta \lambda_{0}$. Le faisceau est ensuite pulsé par un chopper de Fermi qui comporte un collimateur et laisse passer les neutrons lents en premier. Dans une même bouffée, cela introduit un certain délai entre les trois faisceaux. Les trois faisceaux sont finalement refocalisés en temps au niveau des détecteurs: les neutrons diffusés par la plus courte longueur d'onde $\left(\lambda_{0}-\delta \lambda_{0}\right)$ rattrapant ceux diffusés par la plus grande $\left(\lambda_{0}+\delta \lambda_{0}\right)$.

Les trois monochromateurs sont focalisants verticalement permettant ainsi un gain de flux au détriment de la résolution en $Q$, c'est pourquoi le plus petit angle de diffusion est de $10 \mathrm{deg}$.

Le spectromètre secondaire est sous Hélium. Les détecteurs à $\mathrm{He}^{3}$ sont placés à $2.48 \mathrm{~m}$ de l'échantillon pour des angles de diffusion compris entre 10 et $114 \mathrm{deg}$. La résolution en énergie est de $50 \mu \mathrm{eV}$ a $5.9 \AA$ et $170 \mu \mathrm{eV}$ à $4.1 \AA \AA$.

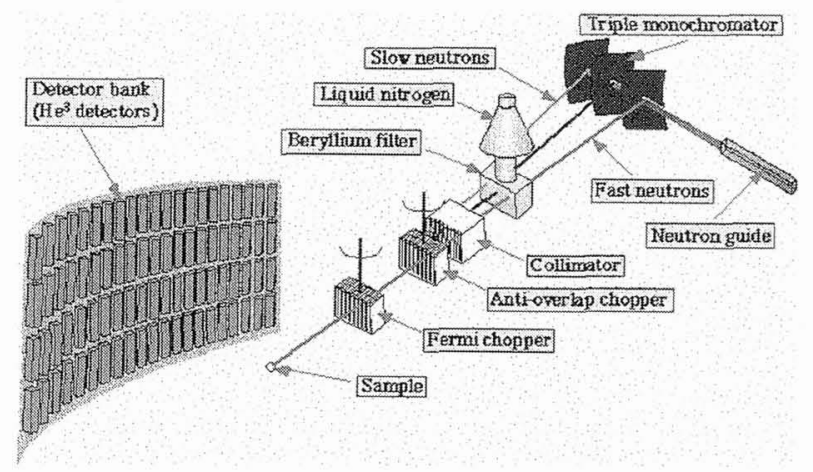

Figure 5: Spectromètre IN6.

\section{4 spectromètre IN4 (ILL)}

Le spectromètre IN4 est construit sur une source de neutrons thermiques permettant d'accèder a des transferts d'énergie plus élévés que sur IN5, IN6 ou Mibémol (typiquement de $10 \ldots 100 \mathrm{meV}$ ).

Le spectromètre primaire est principalement composé de deux choppers de "bruit de fond", d'un monochromateur à double courbure et d'un chopper de type Fermi:

- Les deux choppers de "bruit de fond" elliminent les neutrons rapides et les rayons gamma. Il sont massifs ( $\sim 10 \mathrm{~cm}$ d'acier en fermeture) et tournent en opposition de phase.

- Le monochromateur focalisant à 4 faces permet de sélectionner une longueur d'onde $\lambda$ entre 0.8 et $3.8 \AA$.

- Le chopper de Fermi transmet une bouffée courte $(10 \ldots 50 \mu s)$ à l'échantillon.

La détection est effectuée à $2 \mathrm{~m}$ de l'échantillon par des tubes à $\mathrm{He}^{3}$ dans la gamme de 13 à 120 deg. de l'angle de diffusion. Un multidétecteur couvrant les petits angles devrait être ajouté prochainement. La résolution en énergie $\Delta E / E$ varie entre $2 \%$ et $5 \%$. Elle est principalement gouvernée par les cristaux monochromateurs.

\subsection{Choix de l'instrument}

Voici une liste non exhaustive des questions à se poser pour évaluer la faisabilité d'une experience de "temps-de-vol" et pour sélectionner l'instrument le plus adapte.

- Quelle résolution en énergie est nécessaire pour mesurer le signal quasi-élastique ou inélastique à observer?

La forme de la fonction de résolution a également de l'importance, surtout pour les mesures de quasiélastique. 


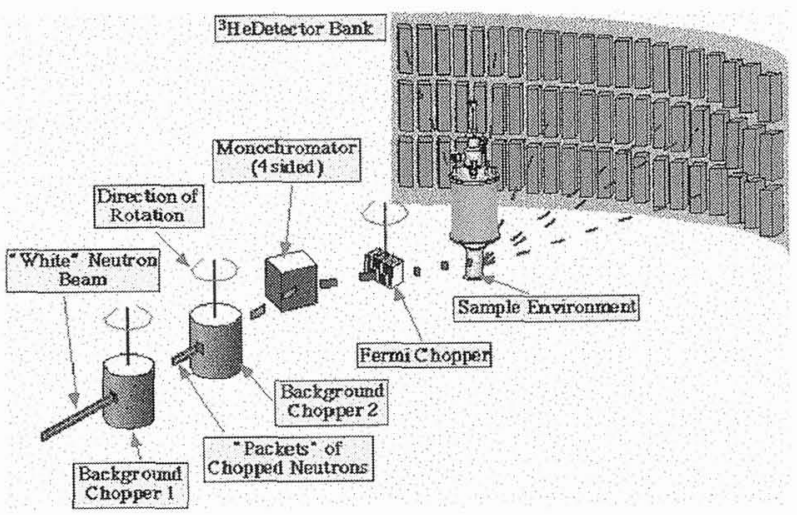

Figure 6: Spectromètre IN4.

- Quel est la dépendance en $Q$ du signal? Cette question peut déboucher sur l'élimination d'une mesure de "temps-de-vol" au profit d'une mesure sur 3-axes. Elle permet aussi de définir la région d'intérêt en $Q$ et de mettre une certaine contrainte sur le choix de la longueur d'onde incidente utilisée. Ceci est important dans le choix de l'instrument: par exemple pour un signal concentré aux petits angles, IN5 est à l'heure actuelle plus approprié puisque qu'il dispose d'un multi-détecteur placé dans le faisceau direct.

- Quel est l'intensité escomptée? Une expérience de "temps-de-vol" se déroule habituellement en quelques jours. Si l'estimation de l'intensité diffusée est faible il faut sans doute préférer un instrument comme IN6 qui dispose d'un flux plus important (si la gamme en Q et la résolution en énergie conviennent au problème étudie). Les mesures en "temps-de-vol" se font habituellement sur des échantillons sous forme de poudre ou liquide et plus rarement sur mono-cristaux. La quantité de matière nécessaire est dépendante du type de propriété étudiée et de la section efficace de diffusion de l'échantillon.

- A quel température doit se faire l'expérience? En effet si la température est petite devant le transfert dénergie à observer, le signal peut seulement être observé par perte d'énergie pour le neutron. Ceci met une certaine contrainte sur le choix de l'énergie incidente et de fait sur la résolution en énergie.

- L'échantillon absorbe-t'il le faisceau de neutrons? Si l'absorption est importante, il faut calculer l'épaisseur pour avoir un bon compromis entre la diffusion et l'absorption. Il faut dans tous les cas calculer l'epaisseur de telle sorte que la diffusion soit inférieure à $10 \%$ pour minimiser les corrections de diffusion multiple. 


\section{TYPE DE MESURES}

Illustration via quelques expériences récentes faites à l'ILL ou à Orphée.

\section{1 densité d'états de phonons dans $A_{1} C_{60}(A=K, R b, C s)$}

\section{H. Schober et B. Renker}

Les cristaux de $C_{60}$ dopés avec des métaux alcalins $A_{1} C_{60}$ (avec $A=K, R b, C s$ ) présentent des diagrammes de phases très riches dus à des réactions chimiques. Ces réactions entrainent la formation de liaisons fortes de covalence entre les molécules de $C_{60}$ (contrairement aux composés de type $A_{3} C_{60}$ et $A_{6} C_{60}$ où la liaison inter-moléculaire se fait via des forces de type Van der Waals ou Coulomb).

Suivant la vitesse de refroidissement deux phases différentes se forment: a) un refroidissement lent conduit à une phase polymère de chaines linéaires b) au contraire, si le refroidissement est suffisamment rapide une phase dimérisée apparait.

La diffusion inélastique des neutrons apportent des informations sur les spectres des excitations struturales (phonons) sur une large gamme d'energie. Dans un systeme comme le $\mathrm{A}_{n} \mathrm{C}_{60}$, il existe typiquement deux domaines d'énergie très différents liés respectivement aux fortes liaisons intra-moléculaire à l'intérieur de la molécule de $\mathrm{C}_{60}$ et aux faibles liaisons inter-moléculaires de type Van der Waals. En présence de polymèrisation ou dimèrisation, cette séparation claire disparait et le gap en énergie entre ces domaines se remplit. La distribution spectrale des excitations est étroitement liée au type particulier de liaisons mises en jeu et a leur force. Ces informations sont obtenues en comparant les donnees expérimentales aux simulations. Par ailleurs, l'évolution en température du spectre des excitations apporte des informations sur le diagramme de phase [1].
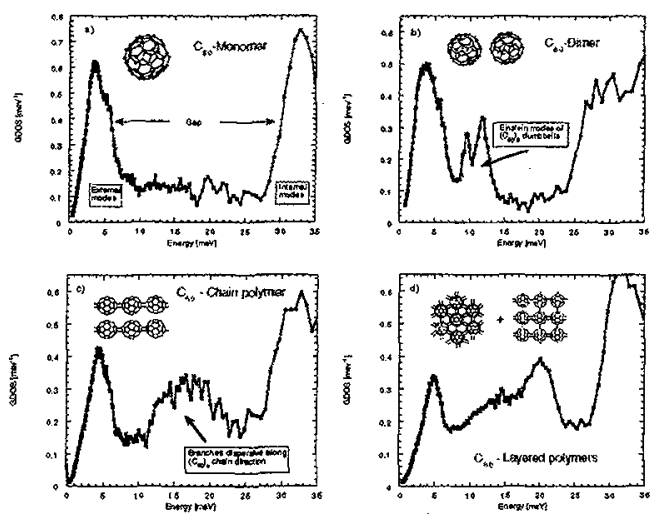

Figure 7: $G(\omega)$ obtenu à partir des mesures faites sur IN6. a) $C_{60}$ a $300 \mathrm{~K}$ dans la phase plastique; b) dimer $\left(C_{60}\right)_{2}$ dans $\mathrm{Rb}{ }_{1} \mathrm{C}_{60}$; c) chaines linéaires de $C_{60}$ polymérisé; d) feuilles de $C_{60}$ polymérisé.

\subsection{Diffusion de l'hydrogène adsorbé dans la zéolithe NaA}

\section{R. Kahn, E. Cohen de Lara, E. Viennet}

Les zeolithes A sont des solides cristallins dont la structure est telle que la maille elementaire (cubique) comporte une cavité presque sphérique d'environ $12 \AA$ de diamètre. Ces cavités communiquent entre elles par des fenêtres circulaires d'environ $4 \AA$ de diamètre dans le cas des zéolithes au sodium (NaA).

La mesure de "temps-de-vol" a êté effectuée sur Mibemol avec un échantillon présentant une quantité d'hydrogène absorbé contrôlée ( $\sim 0.8$ molécule/cavité). Le but était d'étudier la forme du spectre de diffusion quasi-elastique des neutrons $\left(\lambda_{i}=10.58 \AA ; \mathrm{FWHM}=25 \mu \mathrm{eV}\right)$ en fonction de la temperature.

Deux résultats ressortent de cette mesure:

- les molécules d'hydrogène en mouvement ne donnent pas de composante élastique (elastic incoherent structure factor $\mathrm{EISF}=0$ ) impliquant un mouvement de translation à grande distance. 
- la composante quasi-élastique est lorentzienne $S(Q, \omega)=\Delta(Q) / \omega^{2}+(\Delta(Q))^{2}$. La variation de $\Delta$ avec $Q$ (figure de gauche) est bien repressentee par un modele de sauts aléatoires, isotropes en direction:

$$
\Delta(Q)=\frac{1}{\tau_{0}}\left[1-\frac{1}{1+Q^{2} l_{0}^{2}}\right]
$$

La distance moyenne de saut $l_{0} \sim 2.5 \AA$ est indépendante de la température.

Enfin, la pente de $\log D$ en fonction de $1 / \mathrm{T}$ (coordonnées d'Arrhénius, figure de droite) donne l'énergie d'activation de ce mouvement ( $240 \mathrm{~K} \sim 2 \mathrm{~kJ} / \mathrm{mole})$, c'est à dire le potentiel d'interaction moyen entre la molécule et les atomes de la paroi de la cavité [2].

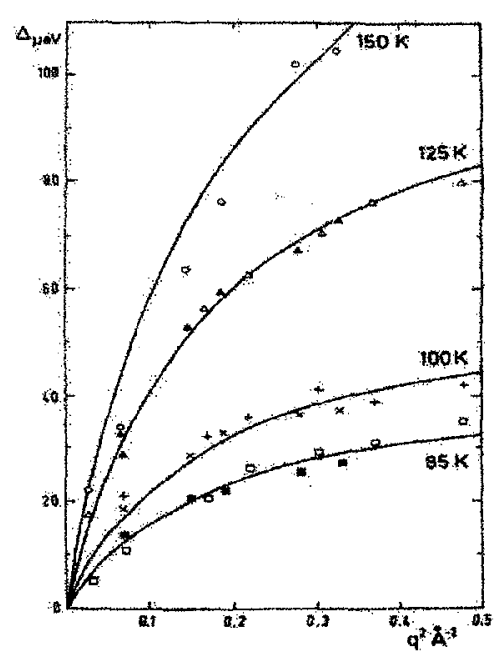

Figure 8a: Largeur en fonction de $\mathrm{q}^{2}$ de la composante quasi-elastique à différentes températures; en trait plein: ajustement avec un modele de diffusion par saut isotrope ( $\Longrightarrow$ liquide).

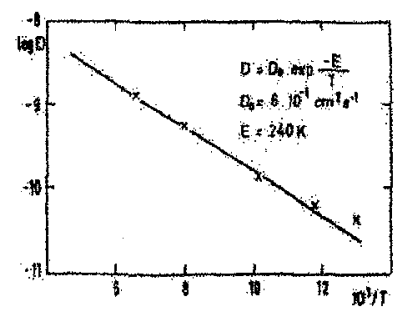

\subsection{Niveaux liés à l'anisotropie magnétique dans la molécule $\mathbf{M n}_{12} \mathrm{O}_{12}$}

\section{Hennion et I. Mirebeau}

Ces amas magnétiques sont synthétisés par chimie moléculaire dans une matrice organique. L'amas ferrimagnétique constitue de $8 \mathrm{Mn}^{3+}(\mathrm{S}=2)$ et de $4 \mathrm{Mn}^{4+}(\mathrm{S}=3 / 2)$ est assimile à un spin unique $\mathrm{S}=10$. L'anisotropie magnétique uniaxiale entrâ̂ne une levée de dégénérescence du niveau fondamental $S=10$ en 11 niveaux correspondant respectivement à $\mathrm{M}=0, \pm 1, \ldots, \pm 10$. L'énergie de ces niveaux est obtenue en diagonalisant un Hamiltonien de spin de la forme suivante:

$$
H=D\left[S_{z}^{2}-1 / 3 S(S+1)\right]+B_{4}^{0} O_{4}^{0}+B_{4}^{4} O_{4}^{4}
$$

avec $O_{4}^{0}=35 S_{z}^{4}-[30 S(S+1)-25] S_{z}^{2}-6 S(S+1)+3 S^{2}(S+1)^{2}$. Le terme transverse $O_{4}^{4}=1 / 2\left(S_{+}^{4}+S_{-}^{4}\right)$ mélange les différents niveaux contrairement aux autres termes. La connaissance de ce terme est cruciale pour la compréhension de l'effet tunnel quantique observé dans ce système à basse température. La diffusion de neutrons est un outil très performant pour ce genre de problème puisqu'elle permet l'observation sans champ magnétique de transitions entre niveaux vérifiants les règles de sélection suivantes: $\Delta S=0, \pm 1$ et $\Delta M=0, \pm 1$. Le calcul de l'intensité magnétique montre que la position et l'intensité des transitions de faible énergie est très sensible à la valeur du coefficient $B_{4}^{4}$ du terme transverse. Ceci permet d'affiner ce coefficient, - important fondamentalement mais de valeur très faible -, par rapport à la valeur déduite des mesures de résonance paramagnétique électronique [3]. 


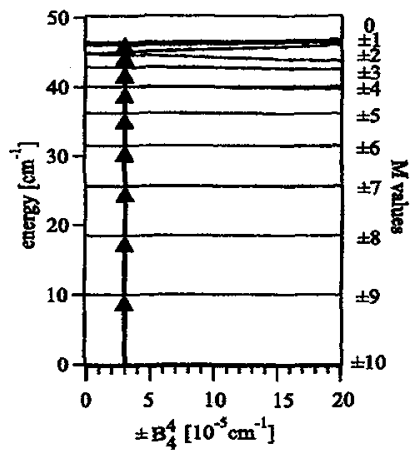

Figure 9a: Etat fondamental de spin $S=10$ de la molécule de $\mathrm{Mn}_{12} \mathrm{O}_{12}$. Les flêches représentent les transitions observables par diffusion de neutrons.

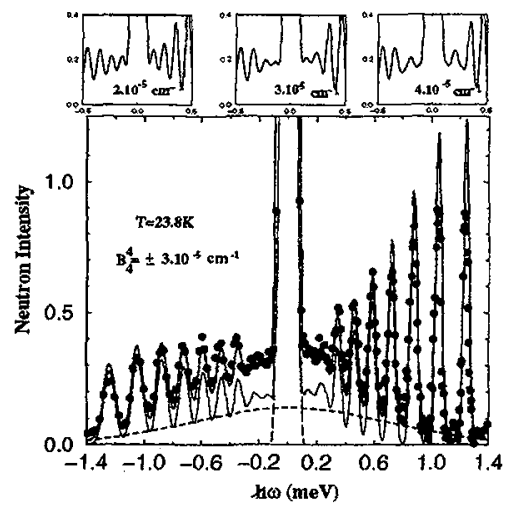

Figure 9b: Spectre obtenu sur IN5 et ajustement pour differentes valeurs du terme de couplage $\mathrm{B}_{4}^{4}$. A $\mathrm{T}=23.8$ $\mathrm{K}$, on observe les transitions en gain (a gauche) et en perte (a droite) d'énergie du neutron.

\subsection{Etude de la dynamique d'un cristal plastique: le Sulfolane.}

\section{J. Combet et M. Bée}

A l'état solide, certains matériaux généralement constitués de molécules symétriques forment une phase plastique à une température de quelques degrés en dessous du point de fusion. Dans cette phase désordonnée l'orientation moléculaire varie de site à site et change avec le temps. L'ordre à grande distance est conservé de façon statistique.

Le Sulfolane $\left(\mathrm{C}_{4} \mathrm{H}_{8} \mathrm{SO}_{2}\right)$ est une molécule de faible symétrie qui possède un moment dipolaire important. Ses propriétés ne sont généralement pas associées à l'existence d'une phase plastique. Des études calorimétriques ont néanmoins démontré l'apparition d'une telle phase désordonnée. Ces résultats ont été confirmés par RMN et par diffraction des rayons X. Le diagramme de phase apparait relativement complexe, la phase plastique se décomposant en deux phases distinctes: les phases I $\left(296<\mathrm{T}<\mathrm{T}_{f}=301.6\right)$ et II $(288<\mathrm{T}<296)$.

Afin de décrire la dynamique des molécules de Sulfolane dans chacune des phases plastiques, des experiences de diffusion quasi-élastique incohérente des neutrons ont été effectuées. Celles-ci ont été réalisées sur le spectrometre "temps-de-vol" IN6 à l'ILL. L'analyse des spectres a été effectuée sur la base de différents modeles géométriques (réorientation isotrope, rotation autour d'un axe...). Un spectre enregistré dans la phase II est présenté sur la figure 10 .

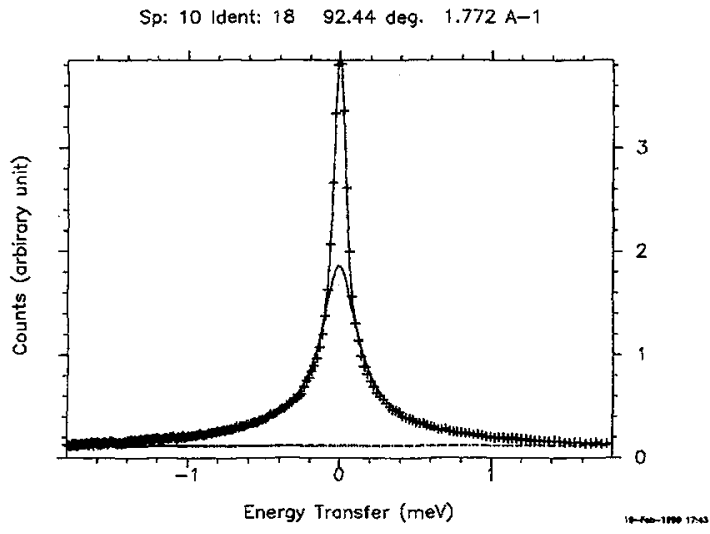

Figure 10: Spectre quasi-élastique (phase II) affiné à l'aide du modéle de rotation uniaxiale. 
Les EISF expérimentaux extraits dans les phases I et II sont représentés sur la figure 11.

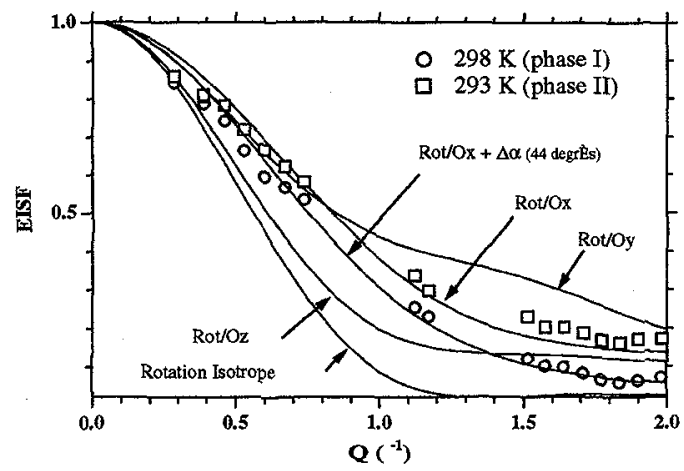

Figure 11: EISF dans les phases I et II.

A T=293 K (phase II), les mouvements peuvent se décrire comme une rotation des molécules autour de leur axe de symétrie (Ox, axe de faible moment d'inertie). A T=298 $\mathrm{K}$ (phase I), le mouvement devient plus complexe. A la rotation uniaxiale s'ajoute désormais une précession de l'axe de symétrie (amplitude d'oscillation 44 degrés). Aucune réorientation isotrope n'a été observée sur l'échelle de temps de l'appareil. Une description complète de la dynamique du système nécessiterait l'utilisation combinée de différents spectromètres (spectrométres "temps-de-vol" / rétrodiffusion...) afin d'étendre la gamme temporelle expérimentale $\left(10^{-10} \mathrm{~s}\right.$ à $\left.10^{-12} \mathrm{~s}\right)[4,5]$.

Annexe 1: Conversions

$$
\begin{aligned}
& 1 \mathrm{meV}=\frac{h c}{e} 10^{5} \mathrm{~cm}^{-1}=8.065478 \mathrm{~cm}^{-1} \\
& 1 \mathrm{THz}=\frac{h}{e} 10^{15} \mathrm{meV}=4.1357 \mathrm{meV} \\
& 1 \mathrm{~K}=\frac{k_{B}}{e} 10^{3} \mathrm{meV}=8.6173510^{-2} \mathrm{meV}
\end{aligned}
$$

Annexe 2: Transformée de Fourier d'une Lorenztienne

$$
S(Q, t)=\int_{-\infty}^{+\infty} S(Q, \omega) \cos (\omega t) d \omega
$$

Transformée de Fourier dans le cas d'une Lorenztienne normalisée $(1 / \pi)$ centrée sur zero:

$$
S(Q, t)=\int_{-\infty}^{+\infty} \frac{1}{\pi} \frac{\Gamma}{\omega^{2}+\Gamma^{2}} \cos (\omega t) d \omega=R e \int_{-\infty}^{+\infty} \frac{1}{\pi} \frac{\Gamma}{\omega^{2}+\Gamma^{2}} \exp (i \omega t) d \omega=\exp (-\Gamma t)
$$

où $\Gamma$ represente la demi-largeur à mi-hauteur.

\section{Remerciements}

Je tiens à remercier J. Combet, C. Czihak, J. Dianoux, M. Hennion, S. Jenkins, R. Kahn, C. Mondelli, I. Mirebeau, A. Murani, H. Mutka, V. Réat, S. Rols et H. Schober pour leur aide lors de l'écriture de ce cours. 


\section{Références}

[1] H. Schober, B. Renker, Neutron News, Vol. 1, No. 1 (1999) 28; H. Schober et coll., Phys. Rev. B 56 (1997) 5937

[2] R. Kahn, E. Cohen de Lara, E. Viennet J. Chem. Phys. 91, (8) (1989) 5097

[3] I. Mirebeau et coll., Phys. Rev. Lett. 83 (1999) 628

[4] J. Combet, M. Bée, D. Djurado, G. D'Azenza, G. Commandeur, Physica B 233 (1997) 95

[5] M. Bee, J. Combet, D. Djurado, J.P. Curtet, J. Dianoux, Physica B 233 (1997) 102

\section{Bibliographie}

- M. Bé "Quasielastic Neutron Scattering: Principles and Applications in Solid State Chemistry, Biology and Material Science" Adam Hilger, 1988.

- "Introduction à la spectromètrie neutronique" CEN-Saclay, Sept.-Oct. 1974. 